Article

\title{
Development of Poly( $\varepsilon$-Caprolactone) Scaffold Loaded with Simvastatin and Beta-Cyclodextrin Modified Hydroxyapatite Inclusion Complex for Bone Tissue Engineering
}

\author{
Jung Bok Lee ${ }^{1,2}$, Ji Eun Kim ${ }^{3}$, Min Soo Bae ${ }^{3}$, Su A Park ${ }^{4}$, Daniel A. Balikov ${ }^{1}$, \\ Hak-joon Sung ${ }^{1,5,9}$, Hoon Bong Jeon ${ }^{6}$, Hun Kuk Park ${ }^{6}$, Soong Ho Um ${ }^{7}$, Kook Sun Lee ${ }^{8}$ \\ and Il Keun Kwon ${ }^{3, *}$ \\ 1 Department of Biomedical Engineering, Vanderbilt University, Nashville, TN 37212, USA; \\ jung.bok.lee@vanderbilt.edu (J.B.L.); daniel.a.balikov@vanderbilt.edu (D.A.B.); \\ hak-joon.sung@vanderbilt.edu (H.S.) \\ 2 Department of Mechanical Engineering, Vanderbilt University, Nashville, TN 37235, USA \\ 3 Department of Maxillofacial Biomedical Engineering, Institute of Oral Biology, School of Dentistry, \\ Kyung Hee University, Seoul 130-701, Korea; sausage14@khu.ac.kr (J.E.K.); bmsbms20@gmail.com (M.S.B.) \\ Department of Nature-Inspired Nanoconvergence Systems, Korea Institute of Machinery and Materials, \\ 156 Gajeongbuk-ro, Yuseong-gu, Daejeon 304-343, Korea; psa@kimm.re.kr \\ 5 Division of Cardiovascular Medicine, Vanderbilt University, Nashville, TN 37235, USA \\ 6 Department of Biomedical Engineering, College of Medicine, Kyung Hee University, 26, Kyungheedae-ro, \\ Dongdaemun-gu, Seoul 130-701, Korea; inwoodkr@gmail.com (H.B.J.); sigmoidus@khu.ac.kr (H.K.P.) \\ 7 School of Chemical Engineering \& SKKU Advanced Institute of Nanotechnology, \\ Sungkyunkwan University, Suwon, Gyeonggi-do 440-746, Korea; Sh.um@skku.ac.kr \\ 8 Department of Oral and Maxillofacial Radiology, College of Medicine, School of Dentistry, \\ Kyung Hee University, Seoul 130-701, Korea; isodent@naver.com \\ 9 Severance Biomedical Science Institute, College of Medicine, Yonsei University, Seoul 120-752, Korea \\ * Correspondence: kwoni@khu.ac.kr; Tel.: +82-10-7154-3000
}

Academic Editors: Esmaiel Jabbari and Frank Wiesbrock

Received: 1 December 2015; Accepted: 5 February 2016; Published: 9 February 2016

\begin{abstract}
In this study, we developed poly(e-caprolactone) (PCL) 3D scaffolds using a solid free form fabrication (SFF) technique. $\beta$-cyclodextrin $(\beta C D)$ was grafted to hydroxyapatite (HAp) and this $\beta C D$ grafted HAp was coated onto the PCL scaffold surface, followed by drug loading through an inclusion complex interaction between the $\beta C D$ and adamantane (AD) or between $\beta C D$ and simvastatin (SIM). The scaffold structure was characterized by scanning electron microscopy (SEM). The release profile of simvastatin in the $\beta$-CD grafted HAp was also evaluated. Osteogenic differentiation of adipose-derived stromal cells (ADSCs) was examined using an alkaline phosphatase activity (ALP) assay. The results suggest that drug loaded PCL-HAp 3-D scaffolds enhances osteogenic differentiation of ADSCs.
\end{abstract}

Keywords: $\beta$-cyclodextrin; hydroxyapatite; poly( $\varepsilon$-caprolactone) 3 -D scaffolds; simvastatin; bone regeneration

\section{Introduction}

In the field of tissue engineering, three-dimensional (3D) porous scaffolds have been fabricated to mimic extra cellular matrix (ECM) found in native tissue for regeneration, restoration, and repair of damaged tissue and organs [1-3]. As their ECM mimetic structures are considered to be critical for successful clinical applications, several rapid prototyping (RP) techniques have been developed 
including selective laser sintering (SLS) [4], stereolithography (SLA) [5], 3D printing [6] and solid free-form fabrication (SFF) [7-9]. In particular, the SFF technique can produce well-controlled, reproducible, scaffold structures such as pore size, porosity, and mechanical properties while varying their internal architecture [9-11].

Synthetic biodegradable polyesters such as poly(lactic-co-glycolic acid) (PLGA), poly(L-lactic acid) (PLLA), and poly( $\varepsilon$-caprolactone) (PCL) have been used as biomaterials in biomedical application due to their excellent biocompatibility and good mechanical properties. Among them, PCL is a thermosensitive polymer with low melting point of about $60^{\circ} \mathrm{C}$ and a glass transition temperature of about $-60^{\circ} \mathrm{C}$, a highly desirable set of temperatures. Its low melting point $\left(60^{\circ} \mathrm{C}\right)$ facilitates the SFF process compared to other polyesters. Furthermore, Several PCL-derived products have also been approved by the Food and Drug Administration (FDA) for clinical applications [12,13]. Although continuous progress has been made so far, PCL has not been applied extensively for bone regeneration, a niche field of tissue engineering that could benefit greatly from utilizing PCL [9,14].

In this study, hydroxyapatite (HAp) was used because it is an attractive material for bone tissue engineering due to its inorganic components boosting affinity for ECM proteins and induction of osteogenic differentiation and mineralization [15-17]. Since $\beta$-cyclodextrin $(\beta C D)$ has been previously used for solubilizing and stabilizing drugs by host-guest type inclusion complexation [18], an inclusion complex system between $\beta C D$ and adamantane $(\mathrm{AD})$ was used to attach HAp onto the surface of the PCL scaffold. Inclusion complex systems have been widely used as a drug loading strategy that forms a bond between ferrocene and cyclodextrin [19]. In solution or in solid state, the cavity of the cyclodextrin generates a hydrophobic environment, thereby changing its physical and chemical interactions with a guest molecule (e.g., drug) [20,21]. For our study, the "driving force" of the complex formation is the fundamental interaction between the $\beta C D$ as the host molecule and $A D$ as the guest molecule. Using this inclusion complex, we produced an HAp-coated PCL scaffold as well as simvastin (SIM)-loaded on HAp that has been known to promote osteogenic differentiation of mesenchymal stem cell via Ras/Smad/Erk signaling derived from bone morphogenic protein (BMP)-2 treatment [22,23]. We used these scaffold templates to examine their osteoconductive/osteoinductive abilities in in vitro human adipose-derived stromal cell (hADSC) and in vivo animal models.

\section{Experimental Section}

\subsection{Materials}

Poly( $\varepsilon$-caprolactone), hydroxyapatite (HAp) nanoparticles (NPs), 3-aminopropyltriethoxysilane (APTES), ascorbic acid, dexamethasone, $\beta$-glycerophosphate (glycerol 2-phosphate disodium salt hydrate), and adamantylamine were purchased from Sigma-Aldrich (St. Louis, MO, USA). $\beta$-cyclodextrin ( $\beta$-CD) was purchased from TCI (TOKYO Chemical Industry Co. Ltd., Tokyo, Japan). Dulbecco's modified Eagle's medium (DMEM), fetal bovine serum (FBS), phosphate buffered saline (PBS), trypsin-EDTA and antibiotics (penicillin-streptomycin) were purchased from Gibco (Rockville, MD, USA). Simvastatin was purchase from Wako (Osaka, Japan). StemPro ${ }^{\circledR}$ Human Adipose-Derived Stem Cells (hADSCs) were obtained from Invitrogen (Carlsbad, CA, USA).

\subsection{Poly(E-caprolactone) (PCL) Scaffold Preparation}

PCL scaffolds were created using a 3D SFF plotting system consisting of a 3-axes machine with a $10 \times 10 \times 10 \mathrm{~cm} x-y-z$ stage, dispenser, nozzle, compression/heat controller, and software/hardware system. PCL pellets were melted in a cylinder at $100{ }^{\circ} \mathrm{C}$ using the heating jacket of the plotting system. The hardware system controlled the scaffold structure by adjusting the pressure, feed rate, and nozzle size. The temperature, pressure, and feed rate were set at $85^{\circ} \mathrm{C}, 650 \mathrm{kPa}$, and $150 \mu \mathrm{m} / \mathrm{s}$, respectively. For the reaction with adamantine, acrylic acid (AAc) was grafted onto the surface of the PCL scaffold. Briefly, the scaffolds were wetted with 70\% ethanol and then immersed in an aqueous AAc solution (10\% in distilled water (DW) with $0.01 \mathrm{M}$ ammonium ferrous sulfate) and exposed to gamma ray 
radiation (10 kGy) from a cobalt-60 source at ambient temperature. Scaffolds were washed with DW three times to remove any unreacted residual monomers or homoplymers. AAc-PCL scaffolds were immersed in adamantylamine (AD, $10 \mathrm{mM}$ )/ ethanol solution for $24 \mathrm{~h}$ with stirring to graft AD onto the PCL scaffold using 1-Ethyl-3-(3-dimethylaminopropyl)-carbodiimide (EDC)/N-hydroxysuccinimide (NHS). Finally, scaffolds were washed with DW three times and freeze dried.

\subsection{Preparation of $\beta$-Cyclodextrin ( $\beta C D)$ Grafted Hydroxyapatite (HAp)}

$\beta C D(10 \mathrm{mmol})$ was dissolved in anhydrous toluene. Then $15 \mathrm{mmol}$ succinic anhydride was added to the solution and the polymers were reacted with stirring at $60{ }^{\circ} \mathrm{C}$ for $12 \mathrm{~h}$ under $\mathrm{N}_{2}$. After the reaction, the product was concentrated by rotary-evaporation. The polymer was then precipitated in diethyl ether. For amino-functionalization of HAp, $5 \mathrm{~g}$ of HAp NPs were immersed in $100 \mathrm{~mL}$ anhydrous toluene containing $10 \mathrm{~mL}$ APTES at $120^{\circ} \mathrm{C}$ under an $\mathrm{N}_{2}$ atmosphere with a reflux condenser for $24 \mathrm{~h}$. The samples were then rinsed for $1 \mathrm{~h}$ in toluene to remove any unreacted silane, and $\beta C D$ was grafted onto the surface of the HAp by a 1-ethyl-3-dimethylaminopropyl carbodiimide- (EDC) mediated reaction between the primary amine groups of the HAp NP surface and the carboxyl group of $\beta C D$. In order to quantify the amine concentration in the HAp surface, the HAp NPs were immersed in $500 \mu \mathrm{M}$ acid orange 7 solution at $\mathrm{pH} 3$ for $3 \mathrm{~h}$ at room temperature and then washed three times with pH 3 DI water. After reaction, the acid orange 7 dye was desorbed by placing the NPs in pH 12 DI water for $30 \mathrm{~min}$. The absorbance was measured at a wavelength of $485 \mathrm{~nm}$ using a spectrophotometer (UV-1650PC, Shimadzu, Japan).

\subsection{Surface Coating of PCL Scaffold with HAp and Simvastatin Loading}

SIM was dissolved in 50\% ethanol at 5 wt \% concentration and then $\beta C D$ grafted HAp NPs ( $3 \mathrm{mg} / \mathrm{mL}$ ) were suspended in the solution. AD-modified PCL scaffolds were immersed in the aforementioned solution and then subjected to ultra-sonication at room temperature for $1 \mathrm{~h}$. SIM was loaded into the $\beta C D$ s of the HAp NPs that were attached to ADs on the surface of the PCL scaffold. After the reaction, the surface of the PCL scaffolds was washed to remove any left over, unloaded HAp NPs and SIM. After 14 days of incubating SIM-loaded samples in a $15 \mathrm{~mL}$ conical tube containing $3 \mathrm{~mL} \mathrm{PBS} \mathrm{(pH} \mathrm{7.4)} \mathrm{at} 37^{\circ} \mathrm{C}$ with orbital agitation at $100 \mathrm{rpm}$, the solution containing released SIM was freeze-dried and redissolved in $1 \mathrm{~mL}$ EtOH. The loading amount of SIM was analyzed by ultraviolet-visible (UV) spectroscopy (UV-1650PC, SHIMADZU, Kyoto, Japan) at a wavelength of $238 \mathrm{~nm}$ and compared to a calibration curve of standard solution at a certain concentration of SIM.

\subsection{Characterization of $\beta C D$-Grafted HAp and PCL Scaffolds}

The surface modified HAp NPs were characterized by thermal gravimetric analysis (TGA, TGA Q5000 IR/SDT Q600, TA Instruments, New Castle, DE, USA) in a scanning range from $25-800{ }^{\circ} \mathrm{C}$ at a constant heating rate of $5^{\circ} \mathrm{C} \mathrm{min}{ }^{-1}$. The surface morphology of HAp-coated PCL scaffolds was analyzed with a scanning electron microscope (SEM, Hitachi S-2300, Tokyo, Japan). Images of samples were taken with $15 \mathrm{kV}$ of an accelerating voltage after sputter-coating with gold. Surface chemical compositions of $\beta C D$-modified HAp and AAc-grafted PCL scaffolds (diameter: $8 \mathrm{~mm}$ and thickness: $3 \mathrm{~mm}$ ) were determined using an X-ray photoelectron spectroscope (XPS, Thermo Fisher Scientific, MA, USA) at a grazing angle of $90^{\circ}$ under high vacuum $\left(<3.1 \times 10^{-9}\right.$ Torr. $)$. Monochromatic aluminum $K \alpha$ X-ray radiation (photoelectron energy $=1486.6 \mathrm{eV}$ ) was used and the wide-scanned XPS spectra were obtained at a pass energy of $187.8 \mathrm{eV}$.

\subsection{Cell Culture}

Human adipose-derived stem cells (hADSCs) were cultured in MesenPRO RSTM medium (MPRO medium, Invitrogen, Carlsbad, CA, USA). To test cell proliferation, hADSCs were seeded on sample PCL scaffolds $(n=4)$ with density of $1 \times 10^{5}$ cells/scaffold and cultured in 48 -well plates in a humidified incubator (Thermo Fisher Scientific, MA, USA) at $37^{\circ} \mathrm{C}$ and $5 \% \mathrm{CO}_{2}$ for 1,4 , and 7 days. 
To examine osteogenic differentiation, hADSCs were seeded onto PCL scaffolds with a density of $1 \times 10^{5}$ cells/scaffold. 1 day after seeding, hADSC-seeded PCL scaffolds were cultured with osteogenic differentiation medium (MesenPRO RSTM medium supplemented with $10 \mathrm{nM}$ dexamethasone, $25 \mu \mathrm{g} / \mathrm{mL}$ L-ascorbic acid, and $10 \mathrm{mM} \beta$-glycerophosphate) for 7, 14, and 21 days. The levels of alkaline phosphatase (ALP) in the differentiated hADSCs cultured on PCL scaffolds were measured using spectroscopy. Briefly, aliquots $(50 \mu \mathrm{L})$ of each sample were incubated in $5 \mathrm{mM} p$-nitrophenol phosphate solution (Sigma, St. Louis, MO, USA) for $30 \mathrm{~min}$ at $37^{\circ} \mathrm{C}$. The level of $p$-nitrophenol production due to the presence of ALP was measured at $410 \mathrm{~nm}$ using a microplate reader (Bio-Rad, Hercules, CA, USA).

\subsection{In Vivo Animal Study}

Male New Zealand rabbits (approximately $2.5 \mathrm{~kg}$ ) were used as an in vivo model to examine bone tissue regeneration. Animal selection, management, and surgery protocol were approved by the Kyung Hee Medical Center Institutional Animal Care and Use Committee, Seoul, Korea (KHMC_IACUC 11-014). Four circular bone defects of $8 \mathrm{~mm}$ in diameter were generated in the cortical bone of a rabbit by pushing a trephine bar through both sides of the inter-parietal suture lines of the cranium without harming the meningeal membrane. PCL scaffolds $(8 \mathrm{~mm}$ in a diameter, $3 \mathrm{~mm}$ in a thickness, $n=3$ per group) were implanted into the defected sites. Each rabbit was anesthetized, sacrificed, and the parietal bone was harvested 6 weeks post implantation. Micro computed tomography $(\mu \mathrm{CT})$ (a SkyScan1173, SKYSCAN, Kartuizersweg 3B 2550, Kontich, Belgium) was used with settings of $130 \mathrm{kV}, 30 \mu \mathrm{A}$ current, and $250 \mathrm{~ms}$ of exposure time to evaluate regeneration of defected bones. The scanned images were reconstructed using NRECON (version 1.6.3.2, Skyscan, Kontich, Belgium, pixel size $=13.85 \mu \mathrm{m}$, angular Step $=0.2^{\circ}$, frame averaging $=5$, beam hardening correction $=40 \%$ ) and 3D images were analyzed using CTAn software (CT Analyser, version 1.14.4.1, Skyscan, Kontich, Belgium), to quantify the total volume of newly formed bone as mean gray value and standard deviation of the region of interest (ROI) from multiplanar reconstructed images.

\subsection{Statistical Analysis}

Statistical analysis was performed using PASW Statistics 18 software (SPSS, Inc., Chicago, IL, USA). All values are expressed as means \pm standard deviations and differences, and $p$-values less than 0.05 were considered statistically significant.

\section{Results and Discussion}

\subsection{Characterization of PCL Scaffolds}

Tremendous efforts have been made to develop ECM mimetic scaffolds in the setting of regenerative medicine [23]. As part of ongoing efforts, we developed HAp-modified PCL scaffolds to represent structural elements of native bone and loaded an osteoinductive drug using an inclusion complex system. In particular, SIM was loaded to recapture osteoinductive effects of the biochemical factors that instruct ECM dynamics in the bone niche (Figure 1). The SEM images of HAp-coated PCL scaffolds are shown in Figure 2. The 3D scaffold structures had highly regular pores of $>600 \mu \mathrm{m}$, porosity of $91.15 \%$ and strands with a thickness of 380-400 $\mu \mathrm{m}$ [24]. After $\beta C D$-modified HAp coating, a homogeneous distribution of HAp NPs along the surface was observed on the AD-modified PCL scaffold surface whereas $\beta C D$-modified HAp NPs were not observed on non-coated PCL scaffolds. 


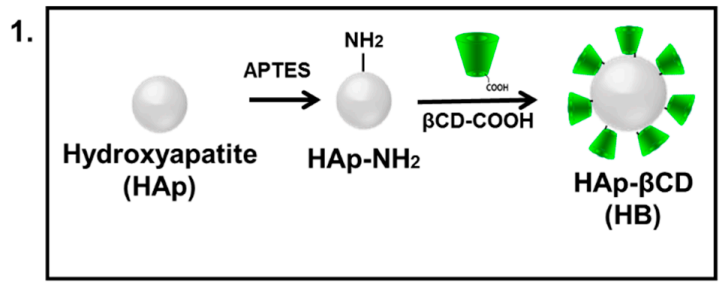

2. PCL scaffold

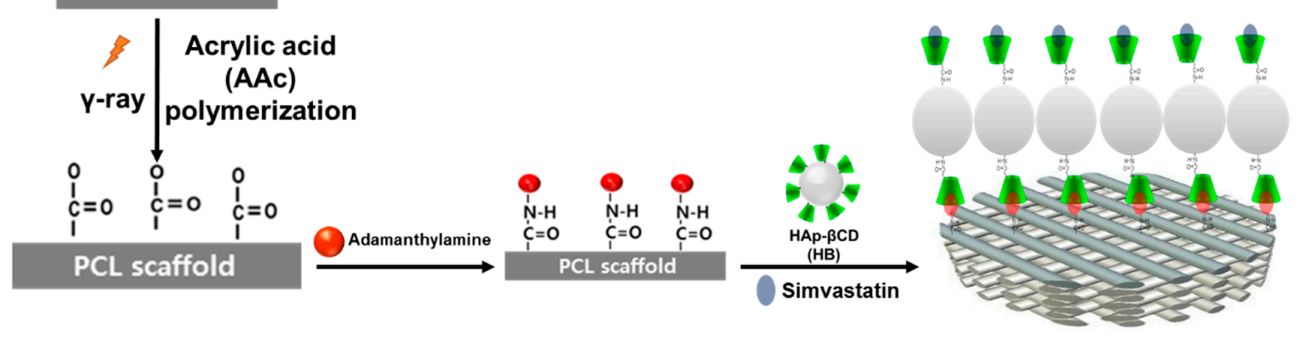

Figure 1. Schematic illustration of hydroxyapatite coated 3D poly( $\varepsilon$-caprolactone) (PCL) scaffolds. (1) Preparation of $\beta$-cyclodextrin ( $\beta C D$ )-grafted hydroxyapatite (HAp) and (2) HAp coating and simvastatin loading on the surface of adamantane (AD)-modified PCL scaffold.

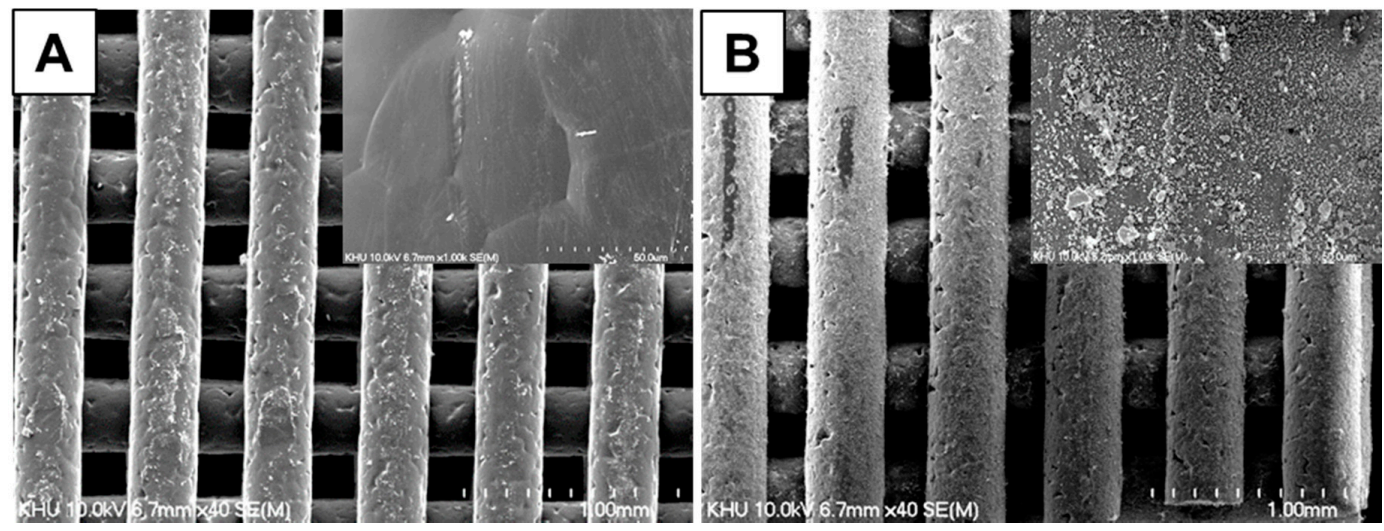

Figure 2. Scanning electron microscopy (SEM) images of $\beta$-cyclodextrin ( $\beta C D$ )-grafted hydroxyapatite (HAp)-coated poly( $\varepsilon$-caprolactone) (PCL) scaffolds (A) without and (B) with adamantane (AD) grafting.

Simulated body fluid (SBF) solution immersion has been commonly used to produce an osteoconductive surface for bone tissue engineering [24,25]. However, its coating procedures, such as using SBF solutions and direct coating of HAp powder, have some disadvantages because SFF requires extended periods of time to successfully work; and the surface structure of the scaffolds can be changed via indiscreet mineral formation, resulting in irreversible alteration of scaffold properties. To overcome these disadvantages, we used $\beta C D$-modified HAp as a coating material and applied this strategy to surface coating of PCL scaffold by an inclusion complex system between $\beta C D$ on the HAp and AD on the PCL scaffold. This reaction produced a homogeneous coating of HAp NPs on the PCL scaffold and enhanced the loading efficiency of the drug [26].

\subsection{Characterization of $\beta C D$-Modified HAp and AD-Modified PCL Scaffold}

To analyze the surface characteristics of $\beta C D$-modified HAp and AD-modified PCL scaffolds, X-ray photoelectron spectroscopy (XPS) was conducted on these samples. After amino-functionalization of HAp and $\beta C D$, the binding energy peaks of Ca2p, P2p, and N1s of HAp NPs were changed (Figure 3) as evidenced by the facts that the Ca2p and P2p peaks of pure HAp were reduced; and the N1s peak was changed from $0.1 \mathrm{eV}$ of HAp to $3.1 \mathrm{eV}$ and $1.6 \mathrm{eV}$ for $\mathrm{HAp}-\mathrm{NH}_{2}$, and $\mathrm{HAp}-\beta C D$, respectively. These results confirmed that the HAp surface was successfully 
amino-functionalized by APTES, leaving $\mathrm{NH}_{2}$ groups for grafting of $\beta C D$ onto the HAp NPs. Table 1 shows the quantified amine concentrations on the amino-functionalization of HAp. The amine concentration of HAp- $\beta C D$ was $3.5 \pm 0.4 \mu \mathrm{mol} / \mathrm{mg}$ and APTES-grafted HAp was $21.9 \pm 2.9 \mu \mathrm{mol} / \mathrm{mg}$. These results showed approximately $18.4 \mu \mathrm{mol} / \mathrm{mg}$ of $\mathrm{NH}_{2}$ groups were generated to graft $\beta \mathrm{CD}$ onto the surface of the HAp NPs. Unexpectedly, some remaining primary amines of the HAp NPs were detected after $\beta C D$ grafting when fluorescent amine was used (data not shown), indicating the need to improve the $\beta C D$ grafting efficiency further. To determine the amount of the grafted $\beta C D$ on the HAp NPs, both non-treated HAp and HAp- $\beta C D$ were analyzed by thermal gravimetric analysis (TGA) at a temperature range of $0-800{ }^{\circ} \mathrm{C}$ (Figure $4 \mathrm{~A}$ ). The TGA curve of both non-treated HAp and HAp- $\beta C D$ showed weight loss at a temperature range of $20-100{ }^{\circ} \mathrm{C}$ as expected given that the scaffolds have some moisture content that can evaporate within this temperature range. Between 300 and $600{ }^{\circ} \mathrm{C}$, weight loss of HAp- $\beta C D$ was observed due to thermal degradation of grafted $\beta C D$, confirming the presence of grafted $\beta C D$.
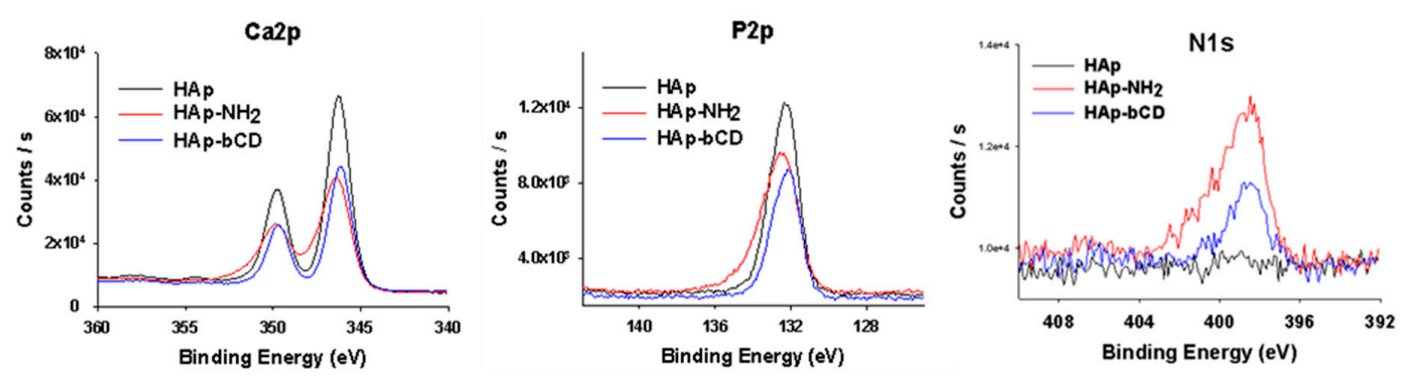

\begin{tabular}{c|c|c|c}
\hline Sample & Ca2p & P2p $_{1}$ & $\mathrm{~N}_{1 \mathrm{~s}}$ \\
\hline HAp & 18.7 & 9.1 & 0.1 \\
\hline HAp-NH2 & 15.0 & 9.1 & 3.1 \\
\hline HAp- $\beta C D$ & 12.1 & 6.42 & 1.62 \\
\hline
\end{tabular}

Figure 3. X-ray photoelectron spectroscopy (XPS) analysis of $\beta$-cyclodextrin $(\beta C D)$ grafted Hydroxyapatite (HAp), 3-aminopropyltriethoxysilane (APTES) grafted HAp, and pure HAp powders.

Table 1. Amine contents of 3-aminopropyltriethoxysilane (APTES)-grafted hydroxyapatite (HAp) and $\beta$-cyclodextrin $(\beta C D)$-grafted HAp.

\begin{tabular}{ccc}
\hline Heading & Hap-NH & Hap- $\beta \mathrm{CD}$ \\
\hline Amine contents $(\mu \mathrm{mol} / \mathrm{mg})$ & $21.9 \pm 2.9$ & $3.5 \pm 0.4$ \\
\hline
\end{tabular}
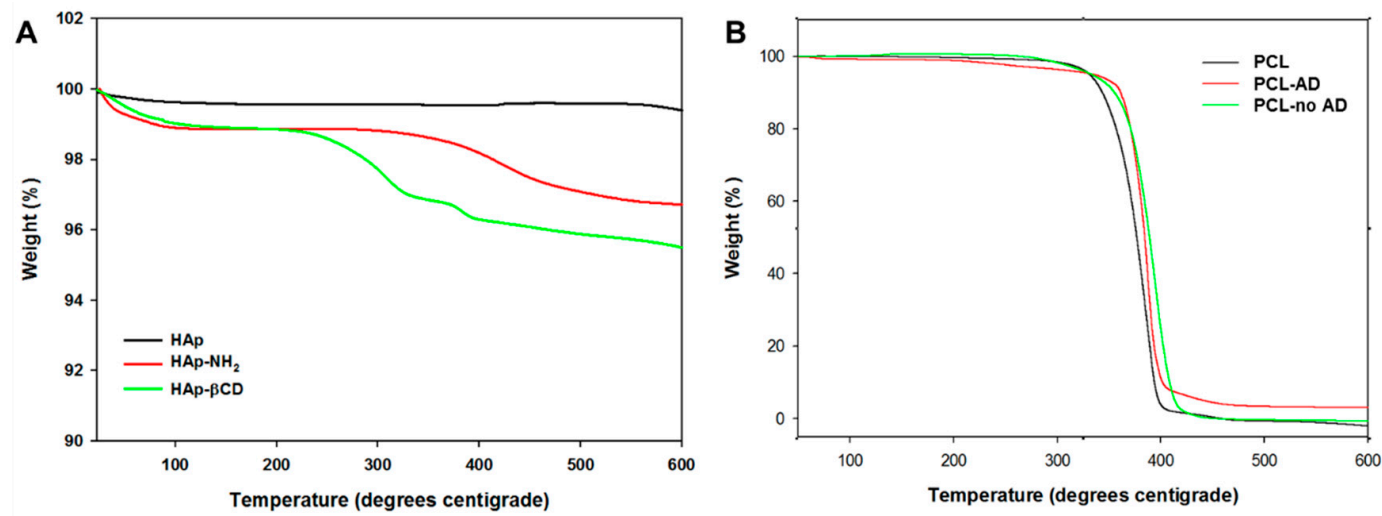

Figure 4. Thermal gravimetric analysis (TGA) of (A) surface-modified hydroxyapatite (HAp) composites and (B) HAp-coated poly( $\varepsilon$-caprolactone) (PCL) scaffolds. 
To determine the degree of AD grafting on to the PCL scaffold surface, acrylic acid (AAc) was grafted onto the surface as the functionalized carboxyl groups could then be stained by toluidine blue (Figure S1). Using the same carboxyl groups, ADs were grafted onto the surface of the PCL scaffold. Figure S2 shows the amount of grafted AD resulting from various AD concentrations. Nearly $100 \%$ of AD in all treatment groups was grafted on the surface of the PCL scaffold regardless of the solution concentration. To measure the amount of coated HAp on the PCL scaffolds, TGA was conducted at a temperature range of $0-600{ }^{\circ} \mathrm{C}$ (Figure 4B). HAp was found to be $7 \mathrm{wt} \%$ whereas $0.7 \mathrm{wt} \%$ was reported for non-AD grafted and $0.2 \mathrm{wt} \%$ for the PCL control.

\subsection{In Vitro Cell Test of PCL Scaffolds}

The initial loading amount of SIM on the HAp- $\beta C D$-coated PCL scaffold was $9.56 \mu \mathrm{g} / \mathrm{mg}$, and $100 \%$ of SIM was released after 14 days (data not shown). Proliferation of hADSCs was measured using the CCK-8 assay kit at 1, 4, and 7 days post culture. Figure 5A shows that there was no observed cytotoxicity among all groups and demonstrated similar proliferation patterns. Figure 5B shows ALP activities of hADSCs on the test PCL scaffolds. Commonly, ALP activity is an early marker of immature osteoblast activity. Moreover, it has been described that ALP cleavage of organic phosphate plays a role in the mineralization of the extracellular collagenous matrix by providing calcium and phosphate ions to generate new formation of the cell-mediated calcium phosphate mineralized matrix $[25,26]$. Elevated levels of ALP activity were noticed on HAp-coated PCL and SIM-loaded HAp-coated PCL scaffolds at day 14 . The ALP activity was significantly higher on the HAp-coated PCL and SIM-loaded HAp-coated PCL scaffolds than the PCL control scaffold at 14 days of cell culture.
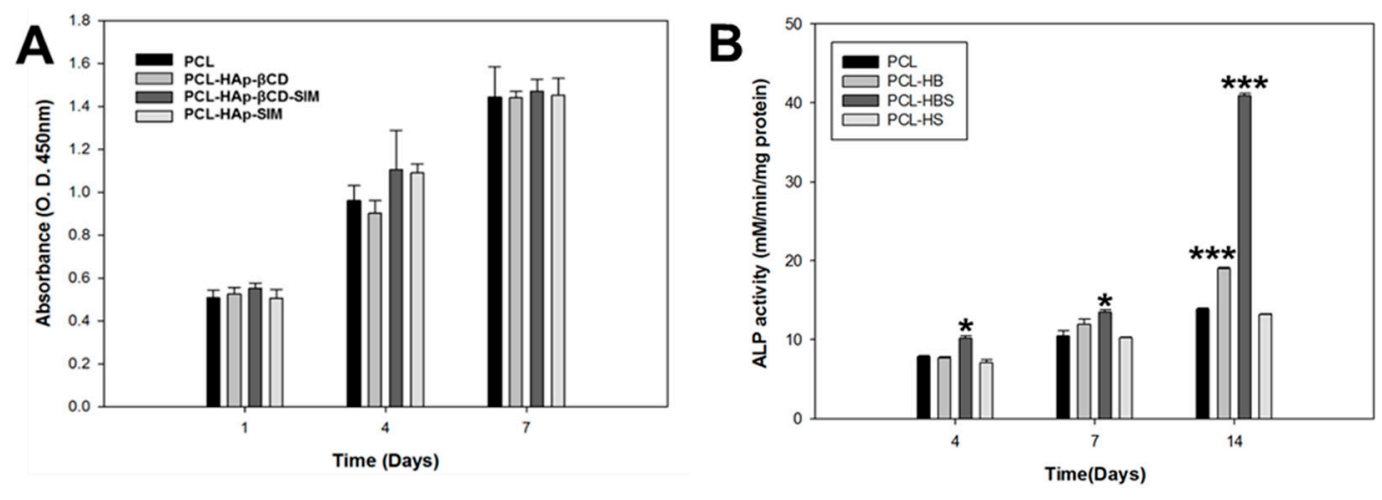

Figure 5. Human adipose-derived stem cell (hADSC) (A) proliferation and (B) alkaline phosphatase (ALP) activity on poly( $\varepsilon$-caprolactone) (PCL) scaffolds.

\subsection{In vivo Animal Study}

The bone defect repair effect of scaffolds was investigated using the rabbit calvarial defect model by in vivo experiments. Figure 6 illustrates the raw $\mu \mathrm{CT}$ images and the corresponding analysis. HAp-coated and SIM-loaded HAp-coated PCL scaffold groups had noticeable increases in bone formation after 6 weeks of implantation as compared to the PCL control group. Using image analysis software, an $8 \mathrm{~mm}$ circular ROI was drawn on the $\mu \mathrm{CT}$ images over the defect site; and the regenerated total bone volume (RBV) was obtained by measuring the mean gray value for this region. The total RBV of all groups had increased over 6 weeks, however the RBV in the SIM-HAp loaded PCL was $22.3 \pm 1.9 \mathrm{~mm}^{3}$ at 6 weeks, which was significantly greater than that observed in the PCL scaffolds groups. Bone regeneration in the defects was found in both HAp-coated and SIM-loaded HAp-coated PCL scaffolds. However the SIM-loaded HAp-coated PCL scaffold group demonstrated more active osteogenesis in the defect area compared with other groups during inplantation periods. The incorporation of SIM and HAp was shown to significantly increase the bone regeneration in vivo. HAp NPs provide a suitable microenvironment for mimicking the inorganic phase of the native tissue 
and SIM has a positive influence on accelerating mineral deposition in vivo. SIM is widely used in clinical lipid-lowering drugs, and it plays a role in promoting new bone formation and regulation by BMP-2 and VEGF expression of osteoblasts [27,28].
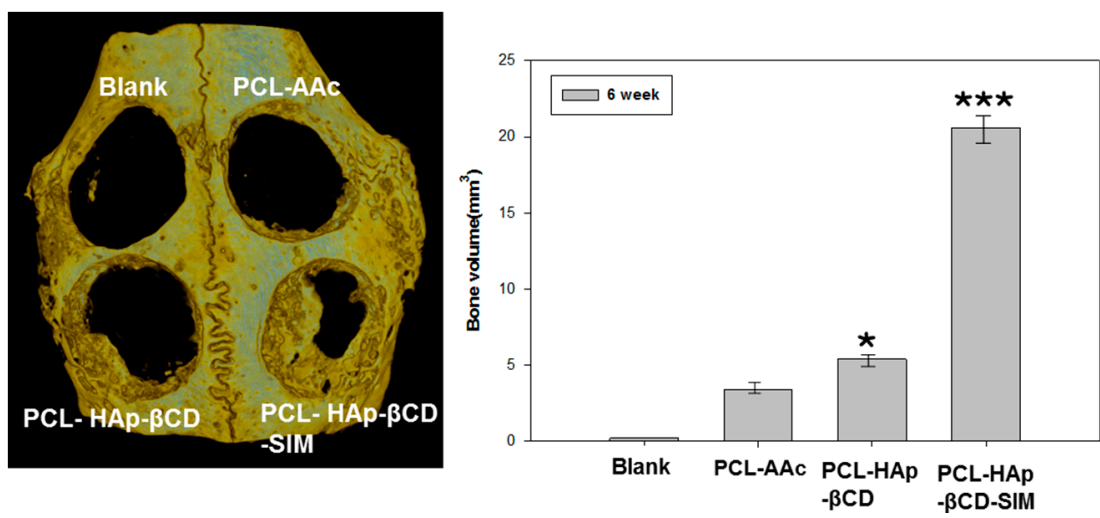

Figure 6. Micro-computerized tomography $(\mu \mathrm{CT})$ scan images of rabbit calvarial defects after 6 weeks of implantation of various poly( $\varepsilon$-caprolactone) (PCL) scaffolds. ${ }^{*} p<0.05$ and ${ }^{* * *} p<0.005$ compared to the PCL-AAc group.

\section{Conclusions}

In this study, we developed PCL scaffolds using a 3D plotting system for bone tissue engineering. We coated PCL scaffolds with $\beta C D$-grafted Hap NPs, followed by SIM loading, using an inclusion complex system. The results indicate that $\beta C D$ grafting enhanced HAp coating to the PCL surface as well as the SIM loading efficiency compared to non-grafted PCL scaffolds. The SIM-loaded PCL scaffold also enhanced the growth and osteogenic differentiation of hADSCs in vitro and bone regeneration in vivo, suggesting the SIM/HAp loaded PCL scaffold as a promising construct for bone tissue engineering.

Supplementary Materials: The following are available online at www.mdpi.com/2073-4360/8/2/49/s1. Figure S1: Schematic illustration of carboxyl group modified PCL scaffold via gamma ray treatment and representative toluidine blue assay stained PCL scaffold, Figure S2: The amount of grafted ADs on the surface PCL scaffolds by treated various concentration of adamantylamine.

Acknowledgments: This work was supported by the Korea Health Technology R\&D Project through the Korea Health Industry Development Institute (KHIDI) funded by the Ministry of Health \& Welfare (HI14C2241).

Author Contributions: Jung Bok Lee and Su A Park developed the scaffold. Jung Bok Lee composed the majority of this manuscript. Jung Bok Lee, Ji Eun Kim, and Min Soo Bae conducted all characterization and in vitro experiments. Daniel A. Balikov, Hak-joon Sung, Hoon Bong Jeon, Hun Kuk Park, Soong Ho Um and Kook Sun Lee aided in troubleshooting of chemical fabrication, guided cell experiments, and edited this manuscript. Il Keun Kwon oversaw the conductance of this entire study and finalized the manuscript.

Conflicts of Interest: The authors declare no conflicts of interest.

\section{References}

1. Langer, R.; Vacanti, J.P. Tissue engineering. Science 1993, 260, 920-926. [CrossRef] [PubMed]

2. Huang, G.Y.; Zhou, L.H.; Zhang, Q.C.; Chen, Y.M.; Sun, W.; Xu, F.; Lu, T.J. Microfluidic hydrogels for tissue engineering. Biofabrication 2011, 3, 012001. [CrossRef] [PubMed]

3. Griffith, L.G.; Naughton, G. Tissue engineering-Current challenges and expanding opportunities. Science 2002, 295, 1009-1014. [CrossRef] [PubMed]

4. Lohfeld, S.; McHugh, P. Laser sintering for the fabrication of tissue engineering scaffolds. In Computer-Aided Tissue Engineering; Liebschner, M.A.K., Ed.; Humana Press: New York, NY, USA, 2012; Volume 868, pp. 303-310. 
5. Melchels, F.P.W.; Feijen, J.; Grijpma, D.W. A review on stereolithography and its applications in biomedical engineering. Biomaterials 2010, 31, 6121-6130. [CrossRef] [PubMed]

6. O'Brien, C.M.; Holmes, B.; Faucett, S.; Zhang, L.G. Three-dimensional printing of nanomaterial scaffolds for complex tissue regeneration. Tissue Eng. Part B Rev. 2015, 21, 103-114. [CrossRef] [PubMed]

7. Sachlos, E.; Czernuszka, J.T. Making tissue engineering scaffolds work. Review: The application of solid freeform fabrication technology to the production of tissue engineering scaffolds. Eur. Cell Mater. 2003, 5, 29-39, discussion 39-40.

8. Kwon, B.-J.; Kim, J.; Kim, Y.H.; Lee, M.H.; Baek, H.S.; Lee, D.H.; Kim, H.-L.; Seo, H.J.; Lee, M.H.; Kwon, S.-Y.; et al. Biological advantages of porous hydroxyapatite scaffold made by solid freeform fabrication for bone tissue regeneration. Artif. Organs 2013, 37, 663-670. [CrossRef] [PubMed]

9. Williams, J.M.; Adewunmi, A.; Schek, R.M.; Flanagan, C.L.; Krebsbach, P.H.; Feinberg, S.E.; Hollister, S.J.; Das, $\mathrm{S}$. Bone tissue engineering using polycaprolactone scaffolds fabricated via selective laser sintering. Biomaterials 2005, 26, 4817-4827. [CrossRef] [PubMed]

10. Rezwan, K.; Chen, Q.Z.; Blaker, J.J.; Boccaccini, A.R. Biodegradable and bioactive porous polymer/inorganic composite scaffolds for bone tissue engineering. Biomaterials 2006, 27, 3413-3431. [CrossRef] [PubMed]

11. Moutos, F.T.; Freed, L.E.; Guilak, F. A biomimetic three-dimensional woven composite scaffold for functional tissue engineering of cartilage. Nat. Mater. 2007, 6, 162-167. [CrossRef] [PubMed]

12. Jiang, C.P.; Chen, Y.Y.; Hsieh, M.F.; Lee, H.M. Solid freeform fabrication and in-vitro response of osteoblast cells of mPEG-PCL-mPEG bone scaffolds. Biomed. Microdevices 2013, 15, 369-379. [CrossRef] [PubMed]

13. Gong, W.; Lei, D.; Li, S.; Huang, P.; Qi, Q.; Sun, Y.; Zhang, Y.; Wang, Z.; You, Z.; Ye, X.; et al. Hybrid small-diameter vascular grafts: Anti-expansion effect of electrospun poly $\varepsilon$-caprolactone on heparin-coated decellularized matrices. Biomaterials 2016, 76, 359-370. [CrossRef] [PubMed]

14. Park, S.; Lee, J.; Kim, Y.; Kim, J.; Lee, J.; Shin, J.-W.; Kwon, I.; Kim, W. Fabrication of biomimetic PCL scaffold using rapid prototyping for bone tissue engineering. Macromol. Res. 2014, 22, 882-887. [CrossRef]

15. Liu, Y.; Wang, G.; Cai, Y.; Ji, H.; Zhou, G.; Zhao, X.; Tang, R.; Zhang, M. In vitro effects of nanophase hydroxyapatite particles on proliferation and osteogenic differentiation of bone marrow-derived mesenchymal stem cells. J. Biomed. Mater. Res. A 2009, 90, 1083-1091. [CrossRef] [PubMed]

16. Dinarvand, P.; Seyedjafari, E.; Shafiee, A.; Jandaghi, A.B.; Doostmohammadi, A.; Fathi, M.H.; Farhadian, S.; Soleimani, M. New approach to bone tissue engineering: Simultaneous application of hydroxyapatite and bioactive glass coated on a poly(L-lactic acid) scaffold. ACS Appl. Mater. Interfaces 2011, 3, 4518-4524. [CrossRef] [PubMed]

17. Shor, L.; Güçeri, S.; Wen, X.; Gandhi, M.; Sun, W. Fabrication of three-dimensional polycaprolactone/ hydroxyapatite tissue scaffolds and osteoblast-scaffold interactions in vitro. Biomaterials 2007, 28, 5291-5297. [CrossRef] [PubMed]

18. Zhang, R.; Ma, P.X. Biomimetic polymer/apatite composite scaffolds for mineralized tissue engineering. Macromol. Biosci. 2004, 4, 100-111. [CrossRef] [PubMed]

19. Liu, Y.; Zhong, R.-Q.; Zhang, H.-Y.; Song, H.-B. A unique tetramer of 4:5 $\beta$-cyclodextrin-ferrocene in the solid state. Chem. Commun. 2005, 2211-2213. [CrossRef] [PubMed]

20. Szejtli, J. Introduction and general overview of cyclodextrin chemistry. Chem. Rev. 1998, 98, 1743-1754. [CrossRef] [PubMed]

21. Hirayama, F.; Uekama, K. Cyclodextrin-based controlled drug release system. Adv. Drug Deliv. Rev. 1999, 36, 125-141. [CrossRef]

22. Mundy, G.; Garrett, R.; Harris, S.; Chan, J.; Chen, D.; Rossini, G.; Boyce, B.; Zhao, M.; Gutierrez, G. Stimulation of bone formation in vitro and in rodents by statins. Science 1999, 286, 1946-1949. [CrossRef] [PubMed]

23. Chen, P.Y.; Sun, J.S.; Tsuang, Y.H.; Chen, M.H.; Weng, P.W.; Lin, F.H. Simvastatin promotes osteoblast viability and differentiation via Ras/Smad/Erk/BMP-2 signaling pathway. Nutr. Res. 2010, 30, 191-199. [CrossRef] [PubMed]

24. Park, S.A.; Lee, S.H.; Kim, W.D. Fabrication of porous polycaprolactone/hydroxyapatite (PCL/HA) blend scaffolds using a 3D plotting system for bone tissue engineering. Bioprocess Biosyst. Eng. 2010, 34, 505-513. [CrossRef] [PubMed]

25. Liu, F.; Malaval, L.; Aubin, J.E. Global amplification polymerase chain reaction reveals novel transitional stages during osteoprogenitor differentiation. J. Cell Sci. 2003, 116, 1787-1796. [CrossRef] [PubMed] 
26. Anderson, H.C.; Sipe, J.B.; Hessle, L.; Dhanyamraju, R.; Atti, E.; Camacho, N.P.; Millan, J.L. Impaired calcification around matrix vesicles of growth plate and bone in alkaline phosphatase-deficient mice. Am. J. Pathol. 2004, 164, 841-847. [CrossRef]

27. Liu, C.; Wu, Z.; Sun, H.-C. The effect of simvastatin on mRNA expression of transforming growth factor- $\beta 1$, bone morphogenetic protein- 2 and vascular endothelial growth factor in tooth extraction socket. Int. J. Oral Sci. 2009, 1, 90-98. [CrossRef] [PubMed]

28. Pagkalos, J.; Cha, J.M.; Kang, Y.; Heliotis, M.; Tsiridis, E.; Mantalaris, A. Simvastatin induces osteogenic differentiation of murine embryonic stem cells. J. Bone Miner. Res. 2010, 25, 2470-2478. [CrossRef] [PubMed]

(C) 2016 by the authors; licensee MDPI, Basel, Switzerland. This article is an open access article distributed under the terms and conditions of the Creative Commons by Attribution (CC-BY) license (http:/ / creativecommons.org/licenses/by/4.0/). 